\title{
Validation of Models of State-to-State Oxygen Kinetics behind Shock Waves
}

\author{
O.V. Kunova, E.V. Kustova, M.Yu. Melnik, A.S. Savelev \\ Saint-Petersburg State University, St. Petersburg, 199034 Russia \\ iEnergyser@gmail.com
}

\begin{abstract}
State-to-state vibrational-chemical kinetics of a binary mixture of oxygen molecules and atoms behind the shock wave is studied. The processes of vibrational energy exchange are described on the basis of the SSH theory and the forced harmonic oscillator model (FHO). Dissociation of oxygen molecules is simulated using the generalized Treanor-Marrone model (TM) with various values of the parameter $U$ available in the literature. All numerical results are compared with experimental data. It is found that the use of the FHO model in combination with the TM model parameter $U=3 T$ or the modified $U$ parameter proposed by Pogosbekian, Sergievskaya (2018) based on trajectory calculations provides satisfactory agreement when compared with the experiment under conditions with the gas temperature behind the shock front greater than $7000 \mathrm{~K}$. Under conditions with the temperature less than $7000 \mathrm{~K}$, the FHO model in combination with the parameter $U=\infty$ or the modified parameter $U$ proposed by Savelev (2016) give the best agreement when compared with the experiments.
\end{abstract}

Keywords: non-equilibrium kinetics, oxygen, shock waves, dissociation.

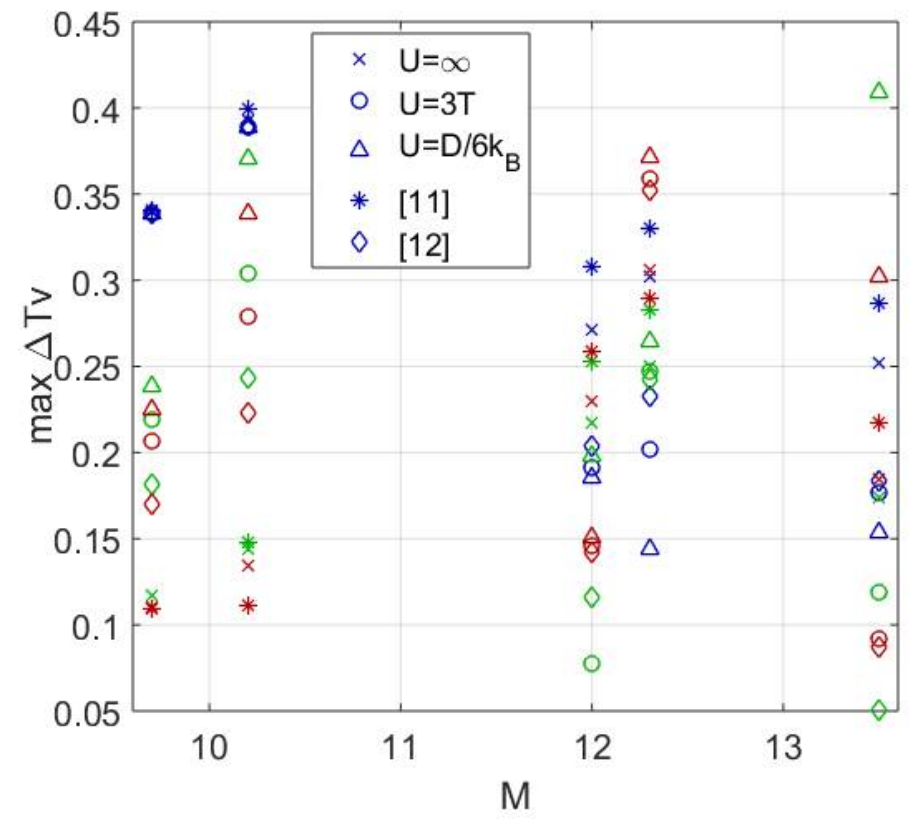

Maximum relative error of vibrational temperature compared with the experimental data, as a function of the incident flow Mach number. Blue markers: SSH model, green: FHO model with single- quantum exchanges, red: FHO model with 1- and 2-quantum exchanges. 


\title{
Валидация моделей поуровневой кинетики кислорода за фронтом ударной волны
}

\author{
О.В. Кунова, Е.В. Кустова, М.Ю. Мельник, А.С. Савельев \\ Санкт-Петербургский государственный университет, \\ Россия, Санкт-Петербург, 199034, Университетская наб., 7/9 \\ iEnergyser@gmail.com
}

\begin{abstract}
Аннотация
В работе исследуется поуровневая колебательно-химическая кинетика смеси молекул и атомов кислорода за фронтом ударной волны. Процессы колебательного энергообмена описывались на основе SSH-теории и модели нагруженного гармонического осциллятора (FHO). Диссоциация молекул кислорода моделировалась с помощью обобщенной модели Тринора-Маррона (ТМ) с различными значениями параметра $U$, доступными в литературе. Все численные результаты сравниваются с экспериментальными данными. Обнаружено, что использование модели FHO в сочетании с параметром модели ТМ $U=3 T$ или модифицированным параметром $U$, предложенным в работе Погосбекяна, Сергиевской (2018) на основе траекторных расчетов, дает хорошее согласие при сопоставлении с экспериментом для условий с температурой газа за фронтом ударной волны больше $7000 \mathrm{~K}$. Для условий с температурой меньше $7000 \mathrm{~K}$ модель FHO в сочетании с параметром $U=\infty$ или модифицированным параметром $U$, предложенным в работе Савельева (2016), дают наилучшее согласие при сопоставлении с экспериментом.
\end{abstract}

Ключевые слова: неравновесная кинетика, кислород, ударные волны, диссоциация.

\section{1. Введение}

За фронтом сильной ударной волны переход к статическому равновесию происходит вследствие взаимосвязанных процессов колебательной релаксации и диссоциации. Настоящая работа посвящена исследованию взаимного влияния кинетических процессов, протекающих в релаксационной зоне стационарной ударной волны в смеси $\mathrm{O}_{2} / \mathrm{O}$, и валидации моделей описания данных процессов. Для тестирования моделей обменов колебательной энергией и диссоциации результаты численного моделирования сравнивались с экспериментальными данными [1].

За последние годы было выполнено большое количество исследований взаимного влияния моделей кинетических процессов в бинарных смесях, из которых можно выделить недавние, полученные в рамках многотемпературного подхода $[2,3]$ и с использованием ПСМ метода [4]. В данной работе моделирование эксперимента в ударной трубе осуществлялось на основе поуровневого подхода с учетом возбуждения колебательных степеней свободы. В результате были определены границы применимости исследуемых моделей колебательной и химической кинетики.

\section{2. Поуровневая кинетика}

\section{1. Кинетические процессы. Основные уравнения}

Колебательно-химическая кинетика процессов, происходящих при столкновениях частиц в двухкомпонентной смеси $\mathrm{O}_{2} / \mathrm{O}$, включает обмены колебательной энергией в молекулах кислорода (VV-обмены) 


$$
\mathrm{O}_{2}(i)+\mathrm{O}_{2}(k) \leftrightarrow \mathrm{O}_{2}\left(i^{\prime}\right)+\mathrm{O}_{2}\left(k^{\prime}\right),
$$

переходы поступательной энергии в колебательную (VT-обмены)

$$
\mathrm{O}_{2}(i)+\mathrm{M} \leftrightarrow \mathrm{O}_{2}\left(i^{\prime}\right)+\mathrm{M}, \quad \mathrm{M}=\mathrm{O}_{2}, \mathrm{O},
$$

химические реакции диссоциации и рекомбинации

$$
\mathrm{O}_{2}(i)+\mathrm{M} \leftrightarrow \mathrm{O}+\mathrm{O}+\mathrm{M}, \quad \mathrm{M}=\mathrm{O}_{2}, \mathrm{O}
$$

Здесь $i, k$ - колебательные уровни молекул $\mathrm{O}_{2}$, штрих обозначает новое колебательное состояние молекул после столкновения.

Система уравнений, описывающая течение рассматриваемой смеси в нулевом приближении метода Энскога-Чепмена [5], включает уравнения неразрывности компонент смеси, уравнения баланса импульса и энергии смеси:

$$
\begin{gathered}
\frac{\mathrm{d} n_{i}}{\mathrm{~d} t}+n_{i} \nabla \cdot \mathbf{v}=R_{i}^{V T}+R_{i}^{V V}+R_{i}^{D R}, \quad i=0,1, \ldots l, \\
\frac{\mathrm{d} n_{a}}{\mathrm{~d} t}+n_{a} \nabla \cdot \mathbf{v}=-2 \sum_{i} R_{i}^{D R}, \\
\rho \frac{\mathrm{d} \mathbf{v}}{\mathrm{d} t}+\nabla p=0 \\
\rho \frac{\mathrm{d} E}{\mathrm{~d} t}+p \nabla \cdot \mathbf{v}=0,
\end{gathered}
$$

и дополняется уравнением состояния

$$
p=n k_{B} T
$$

Здесь $n_{i}$ - заселенности колебательных уровней молекул; $n_{a}-$ числовая плотность атомарного компонента смеси; $n$ - общая числовая плотность смеси; $\mathbf{v}$ - макроскопическая скорость; $\rho$ - плотность смеси; $p$ - давление в смеси; $T$ - температура газа; $k_{B}-$ постоянная Больцмана; $E$ - полная энергия единицы массы

$$
\rho E=\frac{3}{2} n k_{B} T+\rho_{m} E_{\mathrm{rot}}+\sum_{i} \varepsilon_{i} n_{i}+\varepsilon_{a} n_{a},
$$

где $\rho_{m}$-плотность молекулярного компонента; $E_{\mathrm{rot}}-$ вращательная энергия единицы объема смеси; $\varepsilon_{i}$ - колебательная энергия $i$-го уровня молекулы; $\varepsilon_{a}-$ энергия образования атомов. Колебательный спектр молекул кислорода моделировался на основе ангармонического осциллятора.

Правые части уравнений (4)-(5) описывают изменение заселенности колебательных уровней молекул и числовой плотности атомов вследствие химических реакций и колебательного энергообмена, и имеют вид

$$
\begin{gathered}
R_{i}^{V V}=\sum_{k} \sum_{i^{\prime}} \sum_{k^{\prime}}\left(n_{i^{\prime}} n_{k^{\prime}} k_{i^{\prime} i}^{k^{\prime} k}-n_{i} n_{k} k_{i i^{\prime}}^{k k^{\prime}}\right), \\
R_{i}^{V T}=\sum_{\mathrm{M}} n_{\mathrm{M}} \sum_{i^{\prime}}\left(n_{i^{\prime}} k_{i^{\prime} i}^{\mathrm{M}}-n_{i} k_{i i^{\prime}}^{\mathrm{M}}\right), \quad \mathrm{M}=\mathrm{O}_{2}, \mathrm{O}, \\
R_{i}^{D R}=\sum_{\mathrm{M}} n_{\mathrm{M}}\left(n_{a}^{2} k_{i, r e c}^{\mathrm{M}}-n_{i} k_{i, d i s}^{\mathrm{M}}\right), \quad \mathrm{M}=\mathrm{O}_{2}, \mathrm{O}
\end{gathered}
$$


Здесь $k_{i i^{\prime}}^{k k^{\prime}}$ и $k_{i^{\prime} i}^{k^{\prime} k}-$ коэффициенты скорости прямых и обратных $V V$-обменов, $k_{i i^{\prime}}^{\mathrm{M}}$ и $k_{i^{\prime} i}^{\mathrm{M}}-$ коэффициенты скорости прямых и обратных $V T$-обменов при столкновении с частицей сорта $\mathrm{M}, k_{i, d i s}^{\mathrm{M}}$ и $k_{i, r e c}^{\mathrm{M}}-$ поуровневые коэффициенты скорости диссоциации и рекомбинации, соответственно, при столкновении с частицей сорта М.

\section{2. Модели колебательной и химической кинетики}

В настоящей работе для расчета коэффициентов скорости колебательного энергообмена использовались формулы обобщенной SSH-теории $[6,7]$ и модель нагруженного гармонического осцилляторы (FHO), предложенная в [8]. Исследовались одноквантовые переходы энергии $\left(i^{\prime}=i \pm 1, k^{\prime}=k \pm 1\right)$, а также были выполнены расчеты с учетом двухквантовых обменов $\left(i^{\prime}=i \pm 2, k^{\prime}=k \pm 2\right)$ с использованием модели FНО.

Коэффициенты скорости диссоциации рассчитывались на основе обобщенной для поуровневого подхода [9] модели Тринора-Маррона [10]

$$
k_{i, d i s}^{\mathrm{M}}=Z_{i}^{\mathrm{M}} k_{d i s, e q}^{\mathrm{M}}(T), \quad Z_{i}^{\mathrm{M}}=\frac{Z_{v i b r}(T)}{Z_{v i b r}(-U)} \exp \left(\frac{\varepsilon_{i}}{k_{B}}\left(\frac{1}{T}+\frac{1}{U}\right)\right),
$$

где $Z_{v i b r}(T)=\sum_{i} \exp \left(-\frac{\varepsilon_{i}}{k_{B} T}\right)$ - колебательная статистическая сумма; $k_{d i s, e q}^{\mathrm{M}}-$ равновесный коэффициент скорости диссоциации молекулы при столкновении с частицей сорта М.

Расчеты проводились с различными параметрами модели диссоциации $U$ : рассматривались как традиционно использующиеся значения $U=\infty, D / 6 k_{B}, 3 T$ ( $D$ - энергия диссоциации молекул), так и недавно предложенные модификации $[11,12]$, полученные на основе траекторных расчетов.

\section{3. Результаты численных расчетов. Сравнение с экспериментом}

Решение системы уравнений (4)-(7) осуществлялось для условий эксперимента [1]. Условия перед ударной волной представлены в таблице 1, газ до ударного фронта состоял только из молекулярного кислорода.

\section{Таблииа 1}

\section{Условия в набегающем потоке}

\begin{tabular}{|c|c|c|c|}
\hline No. & $p_{\infty}$, Topp & $M_{\infty}$ & $T_{\infty}, \mathrm{K}$ \\
\hline 1 & 2 & 9.66 & 278 \\
\hline 2 & 1 & 10.15 & 308 \\
\hline 3 & 1 & 12.03 & 297 \\
\hline 4 & 1 & 12.3 & 310 \\
\hline 5 & 0.8 & 13.46 & 299 \\
\hline
\end{tabular}

На рис. 1 и 2 представлена зависимость температуры газа $T$ и колебательной температуры молекул смеси $T_{v}$ от времени в сравнении с экспериментальными данными. Для вычисления коэффициентов скорости $V T$ и $V V$-обмена использовалась модель нагруженного гармонического осциллятора (FHO) с учетом только одноквантовых переходов. Коэффициенты скорости диссоциации были рассчитаны с пятью различными значениями параметра $U$ обобщённой модели Тринора-Маррона (ТM). Колебательная температура в поуровневом приближении вычисляется по формуле $T_{v}=\varepsilon_{1} /\left(k_{B} \ln \left(n_{0} / n_{1}\right)\right)$ и зависит от заселенности нулевого и первого колебательного уровня. 
Сравнение численного моделирования течения за ударной волной показало, что для чисел Маха $12 \div 13$ лучшее согласие температуры газа с экспериментом дает расчет с использованием значения $U=3 T$. Также хорошее согласие показывает модель [12]. Противоположная картина наблюдается для более низких чисел Маха (рис. 1 (б)): хорошее согласие наблюдается при $U=\infty$, наилучшее - при использовании модели [11], в то время как использование остальных параметров $U$ приводит к существенно завышенной температуре газа.
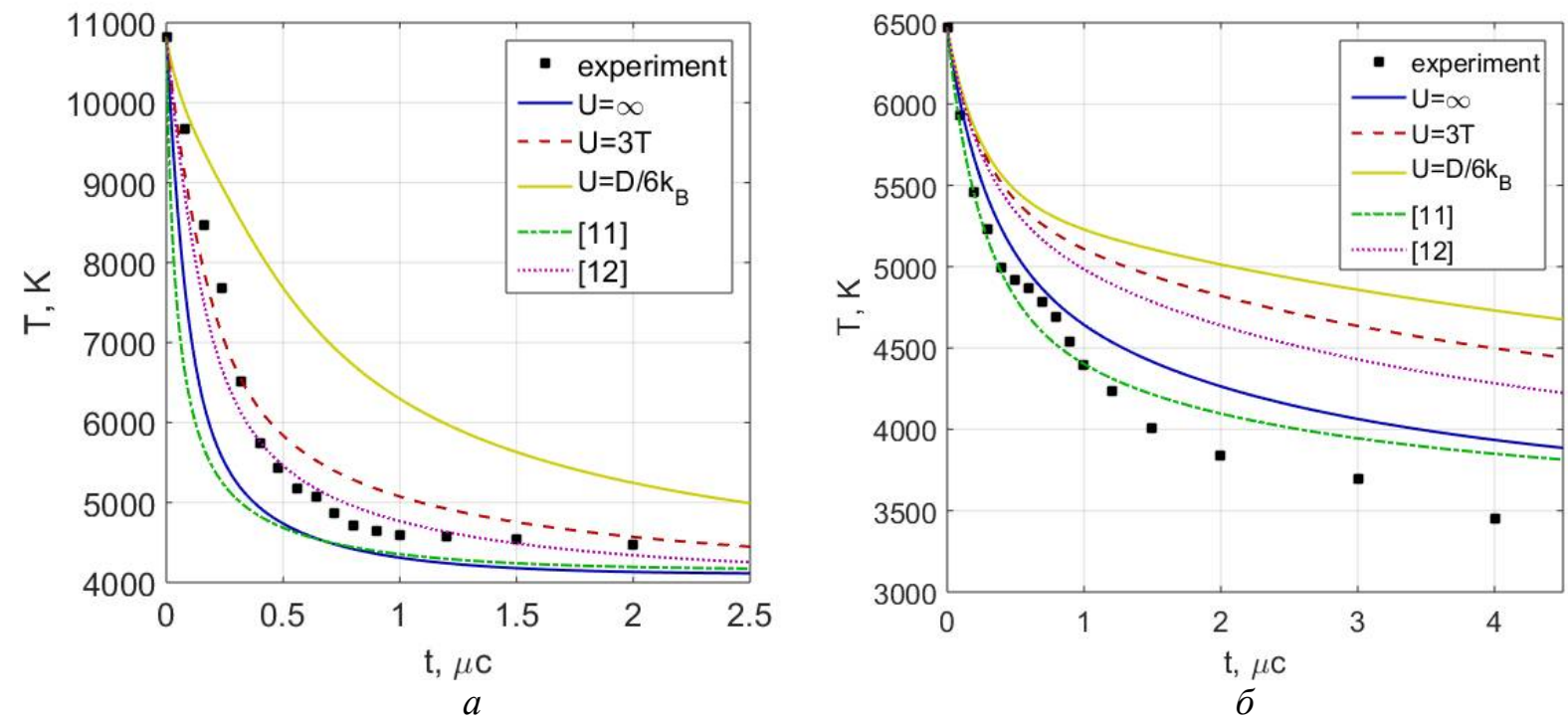

Рис. 1. Изменение температуры газа за фронтом ударной волны с течением времени для начальных условий № 5 ( $а$ ) и № 2 (б)
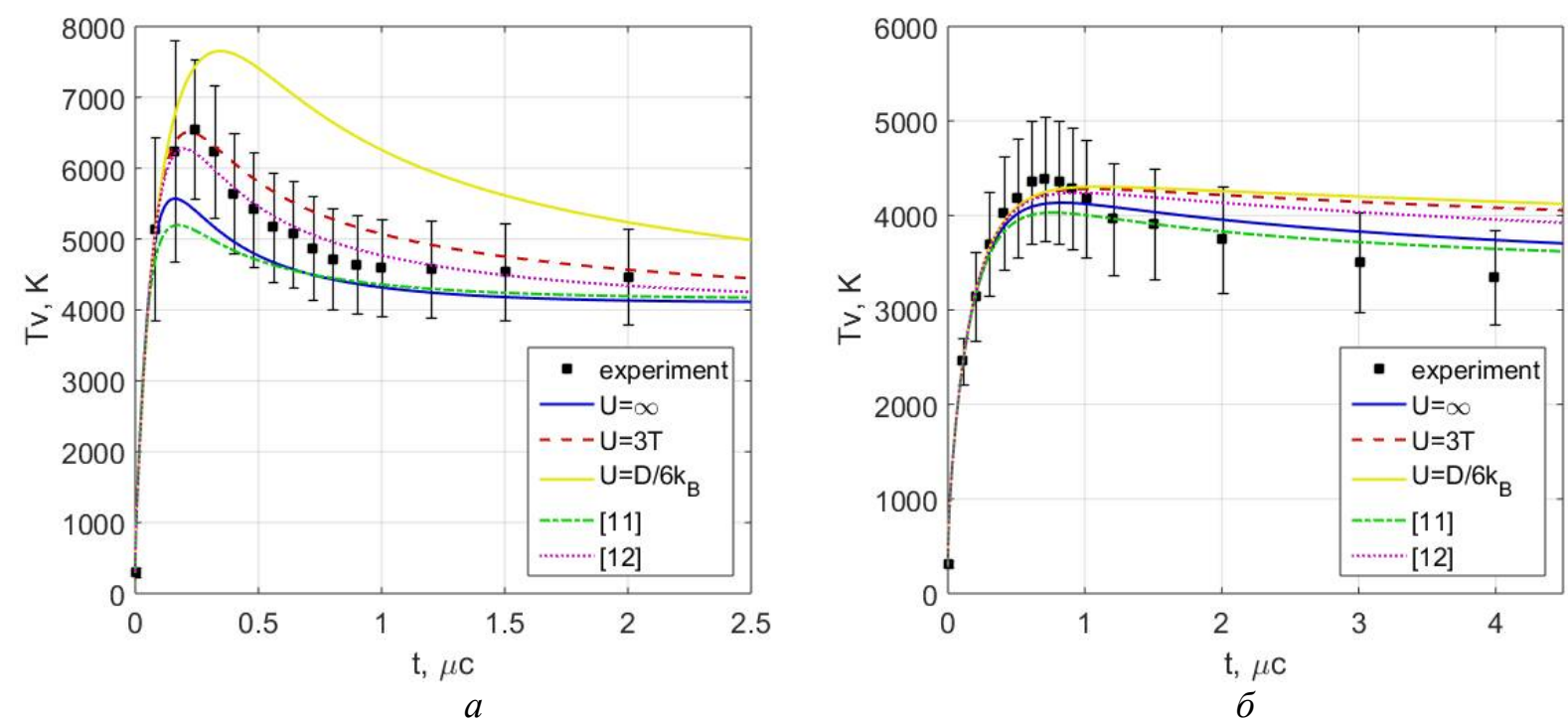

Рис. 2. Изменение колебательной температуры газа за фронтом ударной волны с течением времени для начальных условий № 5 (a) и № 1 (б)

Аналогично для более высоких чисел Маха расчет с использование параметра $U=3 T$ хорошо описывает положение и величину максимума $T_{v}$, а зону уменьшения колебательной температуры - параметр $U$, предложенный в [12] (см. рис. $2(a)$ ). Однако отметим, что кроме случая $U=D / 6 k_{B}$ результаты всех расчетов лежат в пределах экспериментальной погрешности. 
При $M_{\infty}=10.15$ (рис. 2 (б)) все параметры отлично сходятся с экспериментом в зоне колебательного возбуждения газа, зону дезактивации лучше всего описывает модель ТМ с параметром $U$, предложенным в [11].

На рис. 3 представлена эволюция колебательной температуры молекул смеси, полученной с использованием $U=3 T$ в модели TM и моделей $\mathrm{SSH}$ и FHO с учетом 1 и 2-квантовых переходов. Результаты расчетов сравниваются с экспериментальными данными. Видно, что в обоих случаях наилучшее согласие с экспериментом дает модель FHO. При этом в случае с более высоким числом Маха величину и положение максимума $T_{v}$ хорошо описывает расчет с учетом только одноквантовых переходов энергии.

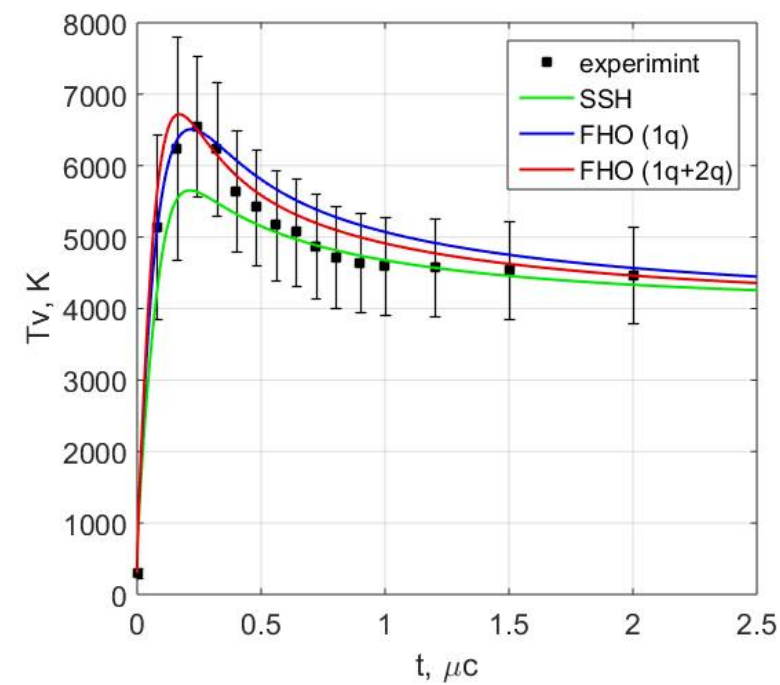

$a$

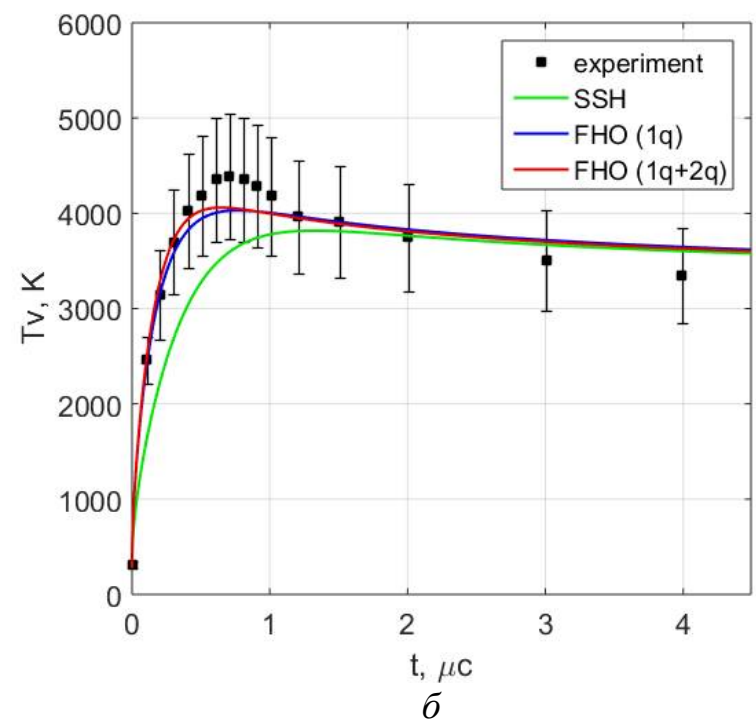

$\sigma$

Рис. 3. Изменение колебательной температуры газа за фронтом ударной волны с течением времени для начальных условий № $5(a)$ и № 1 (б)

На рис. 4 представлено изменение мольно-массовой концентрации молекул кислорода, позволяющее оценить эволюцию состава смеси за ударной волной. Сравнение с экспериментом показывает, что в начале зоны релаксации все параметры модели ТМ кроме $U=D / 6 k_{B}$ существенно переоценивают степень диссоциации. При $t \rightarrow 1 \mu \mathrm{c}$ более точное значение концентрации дает модель ТМ с модифицированным параметром $U[11]$ и $U=\infty$.

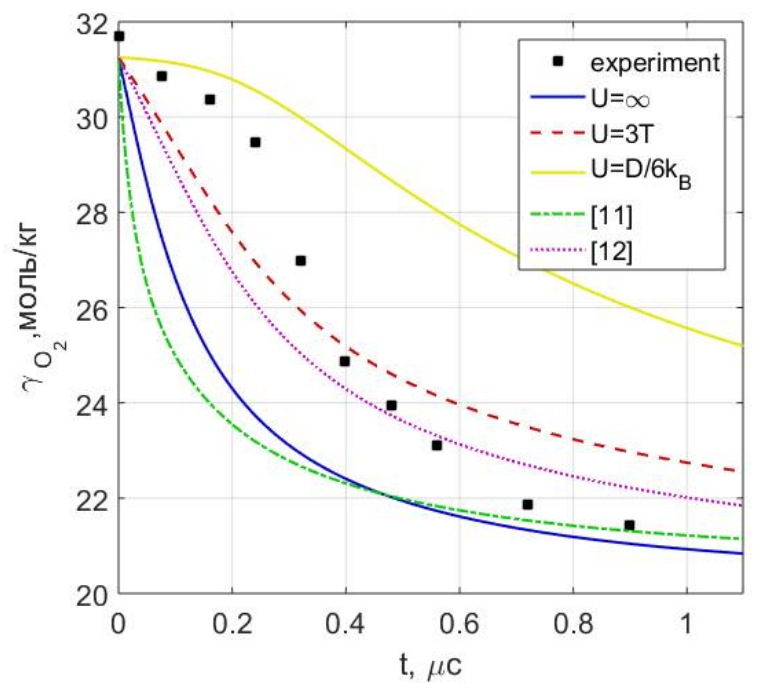

Рис. 4. Изменение мольно-массовой концентрации $\mathrm{O}_{2}$ для начальных условий № 5 
Следует отметить, что экспериментальное начальное значение концентрации молекул несколько превышает все расчетные значения. Возможно, с этим связано не слишком хорошее согласие расчетных концентраций с экспериментально измеренными. При этом согласие с экспериментом по температурам значительно лучше во всех рассматриваемых случаях.

Для оценки границ применимости сочетания моделей колебательных энергообменов и реакций диссоциации-рекомбинации было рассмотрено изменение в зависимости от начальных условий максимального отклонения значений колебательной температуры от экспериментальных данных. На рис. 5 представлены максимальная погрешность численного моделирования для 3-х моделей энергообменов и модели диссоциации ТМ с пятью различными значениями параметра $U$ в зависимости от значения числа Маха.

Для чисел Маха меньше 10.5 наименьшее отклонение от экспериментальной $T_{v}$ показывают модели [11] и $U=\infty$ в совокупности с моделью FHО. Использование модели ТМ с другими параметрами $U$ даёт большее отклонение от эксперимента, существенно завышая колебательную температуру с удалением от фронта (см. рис. 2 (б)).

Для высоких чисел Маха лучшее согласие показывают модели с $U=3 T$ и [12] в сочетании с FHO. Отметим, что в границы экспериментальной погрешности ложится сочетание моделей $\mathrm{SSH}+U=D / 6 k_{B}$. Для остальных рассмотренных параметров $U$ использование SSHтеории при описании энергообменов приводит к большим отклонениям колебательной температуры от экспериментальных значений.

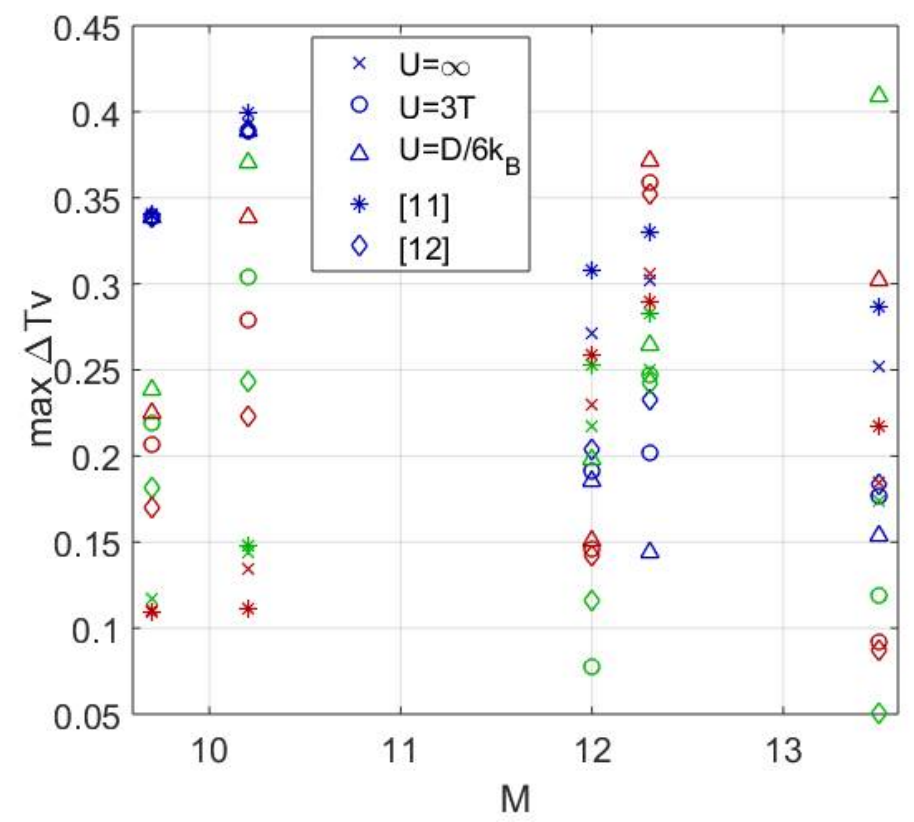

Рис. 5. Максимальное значение относительной ошибки расчета колебательной температуры по сравнению с экспериментальными данными в зависимости от числа Маха набегающего потока. Маркеры синего цвета: модель SSH, зеленого: модель FHO с 1-квантовыми обменами, красного: модель FHO с 1 и 2-квантовыми обменами

Для начальных условий №4 наблюдается иное распределение максимальных отклонений, связанное с сильным отклонением большинства сочетаний моделей от экспериментальных данных в момент времени $t=0.1 \mu \mathrm{c}$.

\section{4. Заключение}

В работе проведено исследование совместного влияния моделей диссоциации и колебательных энергообменов на параметры течения смеси $\mathrm{O}_{2} / \mathrm{O}$ в релаксационной зоне за фронтом ударной волны. Результаты сравнения численного моделирования с существующими 
экспериментальными данными показали, что сочетание модели нагруженного гармонического осциллятора совместно с моделью Тринора-Маррона с параметром $U=3 T$ и модифицированным параметром $U$, предложенным в работе [12], дают хорошее согласие при сопоставлении с экспериментом для условий с температурой газа за ударной волной больше $7000 \mathrm{~K}$, а в сочетании с параметром $U=\infty$ и модифицированным параметром $U$, предложенным в работе [11], дают хорошее согласие при сопоставлении с экспериментом для условий с температурой меньше $7000 \mathrm{~K}$.

\section{Благодарности и ссылки на гранты}

Работа выполнена при поддержке Российского Фонда Фундаментальных Исследований (грант № 18-01-00493) и Санкт-Петербургского государственного университета (НИР № 6.37.206.2016).

\section{Литература}

1. Ibraguimova L.B., Sergievskaya A.L., Levashov V.Y., Shatalov O.P., Tunik Y.V., Zabelinskii I.E. Investigation of oxygen dissociation and vibrational relaxation at temperatures $4000-10800 \mathrm{~K} / / \mathrm{J}$. Chem. Phys. 2013. Vol. 139. N 3. P. 034317.

2. Kustova E., Nagnibeda E., Oblapenko G., Savelev A., Sharafutdinov I. Advanced models for vibrational-chemical coupling in multi-temperature flows // Chem. Phys. 2016. Vol. 464. Pp. 1-13.

3. Shoev G., Oblapenko G., Kunova O., Mekhonoshina M., Kustova E. Validation of vibration-dissociation coupling models in hypersonic non-equilibrium separated flows // Acta Astronautica. 2018. Vol. 144. Pp. 147-159.

4. Sebastião I.B., Kulakhmetov M., Alexeenko A. DSMC study of oxygen shockwaves based on high-fidelity vibrational relaxation and dissociation models // Phys. Fluids. 2017. Vol. 29. N 1. P. 017102.

5. Нагнибеда Е.А., Кустова Е.В. Кинетическая теория процессов переноса и релаксации в потоках неравновесных реагирующих газов. СПб.: Изд-во СПбГУ, 2003. 270 с.

6. Schwartz R.N., Slawsky Z.I., Herzfeld K.F. Calculation of vibrational relaxation times in gases // J. Chem. Phys. 1952. Vol. 20. N 10. Рp. 1591-1599.

7. Физико-химические процессы в газовой динамике / под редакцией Черного Г.Г. и Лосева С.А. / М.: Науч. мир, 2007. Т. 1. 399 с.

8. Adamovich I., Macheret S., Rich J., Treanor C. Vibrational energy transfer rates using a forced harmonic oscillator model // J. Thermophys. Heat Transf. 1998. Vol. 12. N 1. Pp. 57-65.

9. Lordet F., Meolans J., Chauvin A., Brun R. Nonequilibrium vibration-dissociation phenomena behind a propagating shock wave: vibrational population calculation // Shock Waves. 1995. Vol. 4. N 6. Pp. 299312.

10. Marrone P., Treanor C. Chemical relaxation with preferential dissociation from excited vibrational levels // Phys. Fluids. 1963. Vol. 6. N 9. Pp. 1215-1221.

11. Kunova O., Kustova E., Savelev A. Generalized Treanor-Marrone model for state-specific dissociation rate coefficients // Chem. Phys. Lett. 2016. Vol. 659. Pp. 80-87.

12. Погосбекян М.Ю., Сергиевская А.Л. Моделирование реакции диссоциации кислорода в термически неравновесных условиях: модели, траекторные расчеты, эксперимент // Химическая физика. 2018, Т. 37, № 4, С. 20-31. 